\title{
Anthropogenic electromagnetic noise disrupts magnetic compass orientation in a migratory bird
}

Svenja Engels ${ }^{1,2, *}$, Nils-Lasse Schneider ${ }^{1,2,{ }^{*}}$, Nele Lefeldt ${ }^{1,2}$, Christine Maira Hein ${ }^{1,2}$, Manuela Zapka ${ }^{1,2}$, Andreas Michalik ${ }^{1,2}$, Dana Elbers ${ }^{1,2}$, Achim Kittel ${ }^{3}$, P. J. Hore ${ }^{4} \&$ Henrik Mouritsen ${ }^{1,2, \S}$

${ }^{1}$ Institut für Biologie und Umweltwissenschaften, Universität Oldenburg, D-26111

Oldenburg, Germany; ${ }^{2}$ Research Centre for Neurosensory Sciences, University of

Oldenburg, D-26111 Oldenburg, Germany; ${ }^{3}$ Institute of Physics, University of

Oldenburg, D-26111 Oldenburg, Germany; ${ }^{4}$ Department of Chemistry, University of

Oxford, Physical and Theoretical Chemistry Laboratory, Oxford OX1 3QZ, UK.

*These authors contributed equally to this work and are listed alphabetically

${ }^{\S}$ Correspondence and requests for materials should be addressed to henrik.mouritsen@uni-

oldenburg.de

Electromagnetic noise is emitted everywhere humans use electronic devices. For decades, it has been hotly debated whether man-made electric and magnetic fields affect biological processes, including human health ${ }^{1-5}$. So far, no putative effect of anthropogenic electromagnetic noise at intensities below the guidelines adopted by the World Health Organization ${ }^{1-2}$ has withstood the test of independent replication under truly blinded experimental conditions. No effect has therefore been widely accepted as scientifically proven ${ }^{1-6}$. Here we show that migratory birds are unable to use their magnetic compass in the presence of urban electromagnetic noise. When European robins, Erithacus rubecula, were exposed to the background electromagnetic noise present in unscreened wooden huts at the University of Oldenburg campus, they could not orient using their magnetic compass. Their magnetic orientation capabilities reappeared in electrically grounded, aluminium-screened huts, which attenuated 
electromagnetic noise in the frequency range from $50 \mathrm{kHz}$ to $5 \mathrm{MHz}$ by ca. two orders of magnitude. When the grounding was removed or when broadband electromagnetic noise was deliberately generated inside the screened and grounded huts, the birds again lost their magnetic orientation capabilities. The disruptive effect of radiofrequency electromagnetic fields is not confined to a narrow frequency band and birds tested far from sources of electromagnetic noise required no screening to orient with their magnetic compass. These fully double-blinded tests document a reproducible effect of anthropogenic electromagnetic noise on the behaviour of an intact vertebrate.

For more than 50 years, it has been known that night-migratory songbirds can use the Earth's magnetic field to orient spontaneously in their migratory direction when placed in an orientation cage at night in spring and autumn ${ }^{7-8}$. This basic experiment has been independently replicated many times in various locations 9 . We were therefore puzzled to find that night-migratory songbirds tested between autumn 2004 and autumn 2006 in wooden huts on the University of Oldenburg campus $\left(53.1507^{\circ} \mathrm{N}, 8.1648^{\circ} \mathrm{E}\right)$ seemed unable to orient in the appropriate migratory direction. Typical data for European robins are shown in Fig. 1a.

Noting that Ritz et al. ${ }^{10-11}$ had reported the sensitivity of European robins to radiofrequency magnetic fields, in the winter of 2006/2007 we decided to reduce the electromagnetic noise in our test huts by screening them with electrically connected and grounded aluminium plates (Extended Data Fig. 1). The screening left static magnetic fields such as the Earth's completely unaffected, but attenuated the electromagnetic noise inside the huts in the frequency range from about $50 \mathrm{kHz}$ to at least $20 \mathrm{MHz}$ by about 2 orders of magnitude (Fig. 1c-d and Methods). The effect on the birds' orientation capabilities was profound: with the aluminium screens in place, the birds oriented in their normal migratory direction the following spring (2007; Fig. 1b) and in subsequent years (data in references 12-15). When the horizontal 
component of the static magnetic field was rotated $120^{\circ}$ counter-clockwise or when the vertical component was inverted, the birds changed their orientation as expected ${ }^{12-}$ ${ }^{15}$. These observations suggested that, by chance, we could have discovered a biological system that is sensitive to man-made electromagnetic noise in the range up to $5 \mathrm{MHz}$ with intensities well below the guidelines for human exposure proposed by the International Commission on Non-Ionizing Radiation Protection (ICNIRP) and adopted by the World Health Organization ${ }^{1-2}$.

Any report of an effect of low-frequency electromagnetic fields on a biological system should be subjected to particular scrutiny for at least three reasons. First, such claims in the past have often proved difficult to reproduce ${ }^{1-6}$. Second, animal studies are commonly used to evaluate human health risks and have contributed to guidelines for human exposures ${ }^{1-4}$. Third, "seemingly implausible effects require stronger proof $" 16$

Therefore, we systematically conducted a large number of double-blind experiments over the last 7 years to test whether the restored orientation inside the aluminium-screened buildings was really attributable to the reduced exposure to anthropogenic electromagnetic noise. To ensure that our results are reliable, different generations of students independently replicated several key measurements. We also consulted with leading experts to ensure that we very carefully measured the electromagnetic fields experienced by the birds in each of the experimental conditions described below. Electromagnetic fields have magnetic and electric components, and especially in the so-called "near-field" (within a few wavelengths of the source), they must be measured separately.

First, we measured that the aluminium shielding lost its ability to screen anthropogenic electromagnetic noise when the grounding was disconnected (Fig. $2 \mathrm{e}-\mathrm{f})$. We therefore performed a series of experiments in which we tested a group of 
birds alternately in two different, aluminium-screened, wooden huts; one grounded and one left ungrounded. The experimenters were unaware which hut was which. The results were striking: on the days when the birds were tested in a grounded hut, they oriented in their mean northerly migratory direction as expected in spring (Fig. 2a, 2c). By contrast, the same birds were randomly oriented on the days when they were tested in an ungrounded hut (Fig. 2b, 2d). Thus, we could control the orientation of the birds inside the huts by connecting or disconnecting the grounding of the aluminium screens (Fig. 2).

Second, we assessed whether the electromagnetic noise was directly responsible for the disorientation. The birds were tested in the grounded aluminium-screened huts in which they normally orient very well (Fig. 1b, 2a, 2c and data in references 12-15). The birds became disoriented (Fig. 3a) when we introduced broadband electromagnetic noise ranging from $2 \mathrm{kHz}$ up to ca. $9 \mathrm{MHz}$ (Fig. 3d-e, Extended Data Fig. 2) into the huts at magnetic field intensities similar to those measured for the background anthropogenic noise (Fig 1c). To make sure that the observed effect was not simply due to the presence of the signal generator and associated electronics, we repeated these tests under identical conditions but with the output of the signal generator reduced to the lowest possible amplitude (Fig. 3d-e, Extended Data Fig. 2). In this condition, the birds oriented in their migratory direction in spring (Fig. 3b) and re-oriented appropriately when the static magnetic field was rotated $120^{\circ}$ counterclockwise (Fig. 3c). Thus, the disorientation appears to be caused by the electromagnetic noise, and not by the mere presence of the electronics.

Third, we assessed whether the effects are limited to a specific part of the radiofrequency spectrum. To answer this question, we tested European robins inside the grounded, aluminium-screened huts and in the presence of deliberately introduced broadband electromagnetic noise either in the frequency range from ca. $20 \mathrm{kHz}$ to 450 kHz or from ca. $600 \mathrm{kHz}$ to $3 \mathrm{MHz}$ (Fig. 4f-g, Extended Data Fig. 2). As a control, we 
tested the same birds exposed to very low amplitude broadband noise ranging from ca. $2 \mathrm{kHz}$ to $9 \mathrm{MHz}$ (Fig. 3d-e, 4f-g, Extended Data Fig. 2) in which we had observed that the birds could orient (Fig. 3b-c). As expected, the control birds again oriented appropriately (Fig. 4d-e). In contrast, broadband electromagnetic noise in both of the above non-overlapping frequency bands prevented the birds from orienting using their magnetic compass (Fig. 4a-c). Thus, the effects are not limited to one specific frequency or to one part of the radiofrequency spectrum.

The peak magnetic field intensity of the anthropogenic electromagnetic noise at any single frequency measured on typical days around the University of Oldenburg is on the order of $0.1-50 \mathrm{nT}$. The total time-dependent magnetic field, summed over the frequency range $10 \mathrm{kHz}-5 \mathrm{MHz}$, is significantly stronger (on the order of at most 200-1100 nT, see Exteneded Data Table 1), but still much weaker than the Earth's magnetic field (ca. 49,000 nT in Oldenburg). Ritz et al. ${ }^{11}$ reported that the magnetic orientation capabilities of European robins in Frankfurt were disabled by highly directional, monochromatic radiofrequency fields with magnetic field intensities of 15 $\mathrm{nT}$ or more, but not at $5 \mathrm{nT}$ under otherwise identical conditions. Their birds were only disoriented at magnetic intensities below $100 \mathrm{nT}$ when the radiofrequency matched the electron Larmor frequency (1.315 MHz in Frankfurt; $1.363 \mathrm{MHz}$ in Oldenburg), i.e. the resonance frequency of the spin of a free electron interacting with the Earth's magnetic field. Electromagnetic fields similar to those used by Ritz et $a l .{ }^{10-11}$ never occur in natural or urban environments. The anthropogenic electromagnetic noise birds and other living beings experience is not monochromatic, nor is it spatially or temporally coherent (Fig. 1c-d). It has rapidly varying phases and directions and many different frequencies are present simultaneously. The electromagnetic noise we investigated is therefore fundamentally different from the conditions used previously ${ }^{11}$. Furthermore, our birds were never exposed to magnetic fields stronger than $1 \mathrm{nT}$ at $1.315 \mathrm{MHz}$ or $1.363 \mathrm{MHz}$ (Fig.1-5), and two nonoverlapping frequency ranges interfere with the birds' ability to use their magnetic 
compass (Fig. 4). Thus, the disruptive effect on orientation is not limited to a specific resonance frequency. It is caused by electromagnetic fields covering a much broader frequency range and at a much lower intensity (ca. $1 \mathrm{nT}$ at any single frequency) than previously suggested ${ }^{10-11}$. Most importantly, broadband anthropogenic electromagnetic noise omnipresent in industrialized environments can lead to disorientation. These results have several important implications.

First, the present results could have significant consequences for migratory bird conservation. Magnetic compass information is sensed by night-migratory songbirds on the ground and in free flight ${ }^{17-18}$, which mostly takes place at altitudes below $1000 \mathrm{~m} .{ }^{19}$. So, if anthropogenic electromagnetic fields prevent migratory songbirds from using their magnetic compass, their chances of surviving the migratory journey might be significantly reduced, in particular during periods of overcast weather when sun and star compass information is unavailable. Night-migratory songbird populations are declining rapidly ${ }^{20}$, and anthropogenic electromagnetic noise could be a previously neglected contributory factor. Nevertheless, billions of migratory birds do find their way every year. It is therefore pertinent to ask, how localized is the disorienting effect of man-made electromagnetic noise?

We therefore compared the orientation of our robins in the unscreened huts at the university site (Fig. 1a, 5a) with their orientation in an unscreened wooden shelter located ca. $7.5 \mathrm{~km}$ from the university and ca. $1 \mathrm{~km}$ outside the Oldenburg city limit, where the anthropogenic electromagnetic noise was significantly weaker (Fig. 5c-d) and similar in intensity to the electromagnetic noise remaining inside the grounded aluminium-screened huts (Fig. 1c-d, blue trace). In the rural setting, the birds could orient using their magnetic compass in the absence of screening (Fig. 5b). Thus, the disruptive effect of anthropogenic electromagnetic noise on the birds' orientation capabilities appears to be restricted to urban locations where there is typically a high 
usage of electronic devices. Therefore, the effect on wild birds is probably also quite localized.

Second, the results presented here are likely to provide key insight into the mechanism either of the magnetic compass sense $\mathrm{e}^{21-29}$ or of some important process that interferes with the birds' orientation behaviour. The biophysical mechanism that would allow such extraordinarily weak, broadband electromagnetic noise to affect a biological system is far from clear. The energies involved are tiny compared to the thermal energy, $k_{\mathrm{B}} T$, but the effects might be explained if hyperfine interactions in light-induced radical pairs ${ }^{12,21-27}$ or large clusters of iron-containing particles are involved $^{28-29}$. It would be truly remarkable if electromagnetic noise at the intensities studied here could be shown to disrupt the operation of a radical pair sensor by modifying its quantum spin dynamics. To be sensitive to such exceedingly weak magnetic fields, the electron spin-decoherence would have to be orders of magnitude slower than is currently thought possible. This intriguing prospect has attracted the attention of quantum physicists eager to learn lessons from Nature that might ultimately allow more efficient quantum computers to be designed and constructed ${ }^{30}$. Furthermore, we cannot rule out that the birds might be affected by the electric component of the electromagnetic noise, a possibility that has not been considered previously.

Last, but not least, using a double-blinded protocol we have documented a clear and reproducible effect on a biological system of anthropogenic electromagnetic fields much weaker than the current ICNIRP guidelines ${ }^{1-2}$ : the reference levels for general public exposure to time-varying magnetic fields in the relevant frequency band are $6,000 \mathrm{nT}$ at $150 \mathrm{kHz}$ decreasing to $180 \mathrm{nT}$ at $5 \mathrm{MHz}^{1-2}$. The disruptive effects we observe cannot be attributed to power lines (16.7 Hz or $50 \mathrm{~Hz}$ fields) or to mobile phone signals (GHz frequencies) or to any other fields with frequencies below $2 \mathrm{kHz}$ or above $5 \mathrm{MHz}$ because outside this range the electromagnetic noise was of similar 
intensity in all conditions (Fig. 4, Extended Data Fig. 2). Electromagnetic noise in the frequency-band $2 \mathrm{kHz}-5 \mathrm{MHz}$ originates primarily from AM radio signals and from electronic equipment running in university buildings, businesses, and private houses. The effects of these weak electromagnetic fields generated by everyday human activity, however, are striking: they disrupt the function of an entire sensory system in a higher vertebrate.

\section{References}

1. International Commission for Non-Ionizing Radiation Protection. ICNIRP guidelines for limiting exposure to time-varying electric, magnetic, and electromagnetic fields (up to $300 \mathrm{GHz}$ ). Health Phys 74, 494-522 (1998).

2. International Commission for Non-Ionizing Radiation Protection. ICNIRP statement on the "Guidelines for limiting exposure to time-varying electric, magnetic, and electromagnetic fields (up to $300 \mathrm{GHz}$ )". Health Phys 97, 257-258 (2009).

3. World Health Organization. Extremely low frequency fields. Environmental Health Criteria Monograph No.238, http://www.who.int/peh-emf/publications/elf_ehc/en/ (2007).

4. Health Protection Agency. Health effects from radiofrequency electromagnetic fields. http://www.hpa.org.uk/webc/HPAwebFile/HPAweb_C/1317133827077 (2012).

5. Cardis, E. et al. Brain tumour risk in relation to mobile telephone use: results of the INTERPHONE international case-control study. Int J Epidemiol 39, 675-694 (2010).

6. Johansen, C. et al. Cellular telephones and cancer - a nationwide cohort study in Denmark. J Natl Cancer Inst 93, 203-207 (2001).

7. Merkel, F.W. \& Wiltschko, W. Magnetismus und Richtungsfinden zugunruhiger Rotkehlchen. Vogelwarte 23, 71-77 (1965).

8. Wiltschko, W. \& Wiltschko, R. Magnetic compass of European robins. Science 176, 62-64 (1972).

9. Wiltschko, R. \& Wiltschko, W. Magnetic orientation in animals. Springer Verlag, Berlin (1995). 
10. Ritz, T., Thalau, P., Phillips, J.B., Wiltschko, R. \& Wiltschko, W. Resonance effects indicate a radical-pair mechanism for avian magnetic compass. Nature 429, 177-180 (2004).

11. Ritz, T. et al. Magnetic compass of birds is based on a molecule with optimal directional sensitivity. Biophys J 96, 3451-3457 (2009).

12. Zapka, M. et al. Visual but not trigeminal mediation of magnetic compass information in a migratory bird. Nature 461, 1274-1277 (2009).

13. Hein, C.M. et al. Night-migratory garden warblers can orient with their magnetic compass using the left, the right or both eyes. $J R$ Soc Interface 7, 227-233 (2010).

14. Hein, C.M., Engels, S., Kishkinev, D. \& Mouritsen, H. Robins have a magnetic compass in both eyes. Nature 471, E11 (2011).

15. Engels, S., Hein, C.M., Lefeldt, N., Prior, H. \& Mouritsen, H. Night-migratory songbirds possess a magnetic compass in both eyes. PLoS ONE 7, e43271 (2012).

16. Swanson, J. \& Kheifets, L. Biophysical mechanisms: a component in the weight of evidence for health effects of power-frequency electric and magnetic fields. Radiat Res 165, 470-478 (2006).

17. Cochran, W.W., Mouritsen, H. \& Wikelski, M. Migrating songbirds recalibrate their magnetic compass daily from twilight cues. Science 304, 405-408 (2004).

18. Chernetsov, N., Kishkinev, D., Kosarev, V. \& Bolshakov, C.V. Not all songbirds calibrate their magnetic compass from twilight cues: a telemetry study. J Exp Biol 214, 2540-2543 (2011).

19. Bruderer, B. The study of bird migration by radar part 2: major achievements. Naturwiss 84, 45-54 (1997).

20. Sanderson, F.J., Donald, P.F., Pain, D.J., Burfield, I.J. \& Van Bommel, F.P. Longterm population declines in afro-palearctic migrant birds. Biol Conserv 131, 93-105 (2006).

21. Schulten, K., Swenberg, C.E. \& Weller, A. A biomagnetic sensory mechanism based on magnetic field modulated coherent electron spin motion. Z Phys Chem 111, 1-5 (1978).

22. Ritz, T., Adem, S. \& Schulten, K. A model for photoreceptor-based magnetoreception in birds. Biophys J 78, 707-718 (2000).

23. Maeda, K. et al. Chemical compass model of avian magnetoreception. Nature 453, 387-390 (2008).

24. Rodgers, C.T. \& Hore, P.J. Chemical magnetoreception in birds: The radical pair mechanism. Proc Natl Acad Sci USA 106, 353-360 (2009). 
25. Liedvogel, M. \& Mouritsen, H. Cryptochromes - a potential magnetoreceptor: what do we know and what do we want to know? $J R$ Soc Interface 7, 147-162 (2010).

26. Mouritsen, H. \& Hore, P.J. The magnetic retina: light-dependent and trigeminal magnetoreception in migratory birds. Curr Opin Neurobiol 22, 343-352 (2012).

27. Solov'yov, I. A., Domratcheva, T. \& Schulten, K. Separation of photo-induced radical pair in cryptochrome to a functionally critical distance. Sci Rep 4, 3845 (2014).

28. Kirschvink, J.L., Winklhofer, M. \& Walker, M.M. Biophysics of magnetic orientation: strengthening the interface between theory and experimental design. $J R$ Soc Interface 7, 179-191 (2010).

29. Wu, L. Q. \& Dickman, J. D. Neural correlates of a magnetic sense. Science 336, 1054-1057 (2012).

30. Gauger, E. M., Rieper, E., Morton, J. J. L., Benjamin, S. C., and Vedral V. Sustained Quantum Coherence and Entanglement in the Avian Compass. Phys. Rev. Lett. 106, 040503 (2011)

Acknowledgements: We thank Matthias Wuschek, Rohde \& Schwarz, Bundesnetzagentur, and ETS Lindgren for help with measuring the electromagnetic fields, the workshops at the University of Oldenburg, especially Thomas Geiger, for building equipment etc., and a large number of Bachelors, Masters and Ph.D. students for help in conducting the experiments. We are grateful to the following for financial support: Defense Advanced Research Projects Agency (QuBE: N66001-10-1-4061 to PJH and HM), VW-Stiftung (Lichtenberg professorship to HM), DFG (FOR 701 and MO 1408/2-2 to HM), Heinz Neumüller Stiftung (to CMH and SE), BMBF (to HM), the European Research Council (to PJH) and the EMF Biological Research Trust (to PJH).

Author contributions: H.M. and N-L.S designed the study. S.E., N.L., C.M.H., M.Z., A.M. and D.E. performed the experiments. S.E., N.L., C.M.H., M.Z. and H.M. analysed the data. A.K., P.J.H. and NL.S. provided physical insight needed to properly produce and measure the electromagnetic fields. NL.S. and S.E. were in charge of generating the electromagnetic noise. N-L.S. measured the electromagnetic fields. H.M., P.J.H., N-L.S. and S.E. wrote most of the paper. All authors read and commented on the manuscript. 
Author information: Reprints and permissions information is available at www.nature.com/reprints.

The authors declare no competing financial interests. Correspondence and requests for materials should be addressed to henrik.mouritsen@uni-oldenburg.de

Figure 1. Magnetic compass orientation of migratory European robins tested at the University of Oldenburg requires aluminium screening. In unscreened wooden huts, European robins were disoriented (a, spring 2005, $n=21$, mean direction $316^{\circ}$, mean vector length: $r=0.19, P=0.48$ [Rayleigh Test]), but after installing grounded aluminium screens, the birds oriented highly significantly towards North in spring (b, spring 2007, $n=34$, mean direction $356^{\circ} \pm 20^{\circ}$ [95\% confidence interval], $r=0.59, P<0.001$ ). Traces in c-d: anthropogenic electromagnetic noise in the huts before (red) and after (blue) installation of screens. $\mathbf{c}$ and $\mathbf{d}$ show the magnetic and electric components, respectively. In $\mathbf{a}-\mathbf{b}$, each dot indicates the mean orientation of all the tests of one individual bird in the given condition. The dots are colourcoded as in c-d. The arrows show group mean vectors flanked by their $95 \%$ confidence interval limits (solid lines). The dashed circles indicate the minimum length of the group mean vector needed for significance according to the Rayleigh test (inner circle: $P=0.05$; middle: $P=0.01$; outer: $P=0.001$ ). $\mathrm{mN}=$ magnetic North.

Figure 2. Connecting and disconnecting the grounding of the screens turns on and off the birds' magnetic compass orientation capabilities. When the screens were grounded, European Robins oriented significantly in their migratory direction (a: spring 2008, $n=16$, mean direction $341^{\circ} \pm 40^{\circ}, r$ $=0.45, P=0.04)$, whereas they were randomly oriented when the grounding 
was disconnected (b: spring 2008, $n=16$, mean direction $230^{\circ}, r=0.22, P=$ 0.47 ). In another set of identical tests, this pattern repeated itself (c: grounded screens, spring 2008, $n=15$, mean direction $348^{\circ} \pm 41^{\circ}, r=0.48, P=0.03$; d: grounding disconnected, spring 2008, $n=14$, mean direction $290^{\circ}, r=0.12, P$ $=0.82)$. e and f: magnetic and electric field intensities, respectively, when the screens were grounded (blue) or ungrounded (red).

Figure 3. Artificially produced broadband electromagnetic noise disrupts the magnetic compass orientation of birds tested inside the grounded aluminium-screened huts. Broadband noise-modulated electromagnetic fields between $2 \mathrm{kHz}$ and $5 \mathrm{MHz}$ (red traces in 3d-e and $4 \mathrm{~h}-\mathrm{i}$ ) added inside the grounded screens resulted in disorientation of the birds ( $\mathbf{a}$ : autumn $2010, n=22$, mean direction $278^{\circ}, r=0.07, P=0.91$ ). When the same equipment sent out the weakest possible broadband electromagnetic noise (blue traces in $3 \mathrm{~d}-\mathrm{e}$ and $4 \mathrm{~h}-\mathrm{i}$ ), the birds oriented significantly towards North (b: spring 2011, $n=30$, mean direction $354^{\circ} \pm 38^{\circ}, r=0.39, P=0.009$ ) and turned their orientation appropriately when the static magnetic field was rotated $-120^{\circ}$ (c: spring $2011, \mathrm{mN}$ at $240^{\circ}, n=27$, mean direction $253^{\circ} \pm 38^{\circ}$, $r=0.41, P=0.008)$. d: magnetic field intensity. e: electric field intensity.

Figure 4. The disruptive effect of broadband electromagnetic noise on magnetic compass orientation is not limited to a single narrow frequency range. Addition of broadband noise-modulated electromagnetic fields between ca. $20 \mathrm{kHz}$ and $450 \mathrm{kHz}$ (green traces in $\mathbf{f - g}$ ) inside the grounded screens resulted in disorientation of the birds in the normal field (a: autumn 2011, $n=31$, mean direction $306^{\circ}, r=0.24, P=0.17$ ) and in a field turned $-120^{\circ}$ horizontally (b: autumn $2011, n=27$, mean direction $235^{\circ}, r=$ 
$0.03, P=0.96$ ). Broadband fields between ca. $600 \mathrm{kHz}$ and $3 \mathrm{MHz}$ (black traces in $\mathbf{f - g}$ ) also disoriented the birds (c: autumn 2011, $n=30$, mean direction $\left.108^{\circ}, r=0.11, P=0.70\right)$. When the same equipment sent out the weakest possible broadband electromagnetic noise (blue traces in $\mathbf{f - g}$ ), the birds showed appropriately directed magnetic compass orientation (d: autumn 2011, $n=27$, mean direction $258^{\circ} \pm 37^{\circ}, r=0.42, P=0.008$ ), and responded to a $-120^{\circ}$ horizontal rotation of the static field (e: autumn $2011, n=26$, mean direction $107^{\circ} \pm 32^{\circ}, r=0.51, P<0.001$ ). For comparison, the red traces in $\mathrm{f}$ g show the intensity of the strong $2 \mathrm{kHz}$ to $9 \mathrm{MHz}$ broadband noise used for the experiments presented in Fig. 3. f: magnetic field intensity. g: electric field intensity.

Figure 5. In a rural location, European robins show magnetic compass orientation without screening. a: orientation at the university campus (same data as in Fig. 1a). b: orientation at a rural location (spring 2011, $n=28$, mean direction $342^{\circ} \pm 32^{\circ}, r=0.47, P<0.01$ ) where the anthropogenic electromagnetic noise was much weaker (blue traces in c-d) than at the university (red traces in c-d). c: magnetic field intensity. d: electric field intensity.

\section{Methods summary}

Essential methodological information needed for a basic understanding of the text has been woven into the main text at the appropriate places. The online Methods section contains detailed information on: the test subjects, electromagnetic shielding, execution and analysis of behavioural experiments, production and measurement of 
static fields, generation of electromagnetic noise, measurement of time-dependent electromagnetic fields, and blinding procedures.

\section{Methods}

\section{Test Subjects.}

In our study, we tested European robins caught on the campus of the University of Oldenburg, Germany. The birds were housed indoors in individual cages in a windowless room under a light regime simulating the local photoperiod. The tests were performed on the campus of the University of Oldenburg during the spring migratory seasons in 2005 (when we tested 22 birds), 2008 (18 birds) and 2011 (30 birds) and during the autumn migratory seasons in 2010 (24 birds) and 2011 (42 birds). The number of birds caught during the previous migratory seasons and the experimental facilities available for the specific experiment in the given season determined the choice of sample sizes. In addition to these experiments, which were performed specifically for the present study, tests were also conducted by various groups of students in spring $2007^{12}$, spring $2008^{12}$, autumn $2008^{13}$ (tests with garden warblers, Sylvia borin), spring $2009^{12}$, autumn $2009^{14,15}$, autumn $2010^{15}$, and spring $2011^{15}$. These additional experiments, done primarily for other studies that have already been published ${ }^{12-15}$, included tests with control groups which repeatedly confirmed and extended the result presented in Fig. 1, namely that: (a) nightmigratory songbirds orient properly using their magnetic compass in the grounded and screened huts in the unchanged geomagnetic field ${ }^{12-15}$, and (b) they adjust their orientation appropriately when the horizontal component of the static field is rotated by $-120^{\circ 12-15}$. Furthermore, in two previous studies ${ }^{12,15}$ we tested groups of European Robins in the screened and grounded huts while exposing them to a static field the vertical component of which had been inverted, leaving the horizontal component still pointing to the North. In this field, the polarity of the field lines are unchanged and still points towards magnetic North, but the axis of the static field lines is the same as if the static field had been turned $180^{\circ}$ horizontally. Since these robins flipped their orientation ca. $180^{\circ 12,15}$, the birds in the grounded and screened huts were using their standard magnetic inclination compass ${ }^{8,9}$. All animal procedures were approved by 
the Animal Care and Use Committees of the Niedersächsisches Landesamt für Verbraucherschutz und Lebensmittelsicherheit (LAVES, Oldenburg, Germany).

\section{Static magnetic fields.}

Static magnetic fields were produced with double-wrapped, three-dimensional Merritt four-coil systems ${ }^{31}$ with an average coil dimension of $2 \mathrm{~m}$. All experiments were performed within the central space of the coils where the magnetic field homogeneity was better than $99 \%$. Before the beginning of each experiment, the ambient geomagnetic field was measured using a Flux-Gate magnetometer (FVM-400, Meda Inc.) in the centre and at the edges of the experimental volume within which the orientation funnels were placed. Birds were tested in two different static magnetic field conditions: in a magnetic field closely similar to the natural geomagnetic field in Oldenburg (Normal Magnetic Field, NMF) and in a magnetic field of the same strength and inclination as the local geomagnetic field but rotated $120^{\circ}$ counterclockwise in the horizontal plane (Changed Magnetic Field, CMF). To produce the CMF condition, the appropriate currents ran through the two subsets of windings per axis of the three-axial, four-coil Merritt system in the same direction. In the NMF condition, the currents that were needed to produce the CMF condition ran through the two subsets of windings but in opposite directions so that no significant changes (i.e. $<10 \mathrm{nT}$ ) to the geomagnetic field were produced by the coils ${ }^{31}$. The actual fields experienced by the birds under the two magnetic field conditions were as follows (mean \pm standard deviation). NMF condition: $48,900 \mathrm{nT} \pm 150 \mathrm{nT}$; inclination $=67.7^{\circ}$ $\pm 0.6^{\circ}$; horizontal direction $=360^{\circ} \pm 0.1^{\circ} . \mathrm{CMF}$ condition: $49,000 \mathrm{nT} \pm 470 \mathrm{nT}$; inclination $=68.0^{\circ} \pm 1.1^{\circ}$; horizontal direction $=-120^{\circ} \pm 0.5^{\circ}$.

\section{Electromagnetic shielding of experimental huts.}

Most of the experiments were performed inside wooden huts (Extended Data Fig. 1a) placed at the Wechloy (natural sciences) Campus of the University of Oldenburg (Extended Data Fig. 1d) in the city of Oldenburg (population ca. 160,000; Extended Data Fig. 1c). Some of the orientation experiments in spring 2011 took place in an 
unscreened wooden shelter, normally occupied by horses, located in fields ca. $7.5 \mathrm{~km}$ from the university and ca. $1 \mathrm{~km}$ outside the built-up part of the city of Oldenburg (Extended Data Fig. 1c). An earth barrier in the form of a highway bridge foundation was located between the testing location and the city of Oldenburg.

To attenuate time-dependent electromagnetic fields inside the wooden huts, the four walls (including the door) and the roof were covered with $1 \mathrm{~mm}$ thick aluminium sheets, overlapping by at least $20 \mathrm{~mm}$ and bolted together with self-cutting screws every 5-10 cm (Extended Data Fig. 1b). We also tested whether the efficiency of the screens could be improved by adding aluminium sheets on the floor. No significant improvement was found, probably because negligible electromagnetic noise comes from below. Most of the experiments were therefore performed in huts screened on five sides in which the air-circulation was improved and the humidity variability reduced compared to shielding on six sides.

The aluminium walls of this five-sided Faraday cage were interconnected at all times. In the grounded conditions, this aluminium screening assembly was electrically connected at a single location to a single grounding rod with a depth of $8 \mathrm{~m}$. In the ungrounded conditions, the grounding rod was manually disconnected from the aluminium screening assembly. Disconnection of the grounding removed the screening effect of the aluminium shields. In fact, the ungrounded aluminium screens acted as an antenna that slightly increased the magnetic field intensity at some frequencies inside the test chambers compared to the unscreened condition (compare Fig. 1c-d with Fig. 2e-f). The disconnection of the grounding during the critical grounding/ungrounding experiments (Fig. 2) was performed by a member of the lab, who was not involved in the behavioural experiments, and the persons performing and evaluating the experimental results were not aware of the change in conditions until after the completion of the experiments.

All electronic devices were placed outside this cage, disconnected from their protected earths and grounded via the same grounding rod as the Faraday cage. This is necessary because the protected earth from the standard power outlet would act as an antenna and introduce electromagnetic noise into the system. When properly grounded, the shielding attenuated the time-dependent magnetic fields with frequencies up to ca. $20 \mathrm{MHz}$ by approximately two orders of magnitude inside the 
testing chambers. The screening efficiency was estimated by generating electromagnetic noise just outside the chambers while measuring the electromagnetic noise arriving within. The anthropogenic electromagnetic noise observed at the University of Oldenburg is dominated by frequencies below $5 \mathrm{MHz}$. Higher frequency contributions were mostly at or below the detection limit of our equipment and are therefore not shown in Figs 1-5.

\section{Generation of electromagnetic noise.}

To produce electromagnetic noise, a passive loop antenna (ETS Lindgren, Model $6511,20 \mathrm{~Hz}-5 \mathrm{MHz}$ ) was placed vertically under the centre of the central orientation funnel and aligned along the north-south axis $(48 \mathrm{~cm}$ vertically from the centre of the loop to the central funnel).

Broadband electromagnetic noise in the range $2 \mathrm{kHz}$ to $9 \mathrm{MHz}$ was produced by a signal generator (Hewlett Packard, 33120A, 15 MHz Arbitrary Waveform Generator) connected to the antenna using either the maximum output (10 V peak-topeak) for the strong noise condition or the minimum output ( $50 \mathrm{mV} \mathrm{pp}$ ) for the weak noise condition used as a control (the generated noise with the output set to $50 \mathrm{mV} p \mathrm{p}$ was weaker than the measurement limit except for the electric component below 500 kHz, see blue traces in Fig. 3d, 3e and Fig. Extended Data Fig. 2). An alternative to this control would have been to use the "silent shorting" design suggested by Kirschvink et $a l^{28}$. We experimented with this method, but even the shorted condition led to measurably increased electromagnetic fields inside the huts, which is why we chose the control described above.

The band-pass electromagnetic noise $(20 \mathrm{kHz}-450 \mathrm{kHz}$ and $600 \mathrm{kHz}-3 \mathrm{MHz})$ was produced using a vector signal generator (Rohde \& Schwarz, SMBV 100A, 9 $\mathrm{kHz}-3.2 \mathrm{GHz}$ ) connected to the same passive loop antenna.

\section{Measurements of time-dependent electromagnetic fields.}


The magnetic and electric components of the time-dependent electromagnetic fields were measured separately with different antennas connected to a signal analyser (Rohde \& Schwarz, FSV 3 Signal and Spectrum Analyzer 10 Hz-3.6 GHz). All such measurements were performed at a similar time of day as the behavioural experiments, but not while the actual tests were running. This procedure was chosen because we wanted to exclude any possibility that the measurements or measuring equipment could influence in any way the electromagnetic noise fields present while the birds were being tested.

The magnetic component between $10 \mathrm{kHz}$ and $5 \mathrm{MHz}$ was measured with a calibrated passive loop antenna (ETS Lindgren, Model 6511, $20 \mathrm{~Hz}-5 \mathrm{MHz}$ ). The electric component between $10 \mathrm{kHz}$ and $10 \mathrm{MHz}$ was measured with a calibrated active biconical antenna (Schwarzbeck Mess-Electronik, EFS 9218, 9 kHz-300 MHz). The signal analyser was set to 'max hold' and a resolution bandwidth of $10 \mathrm{kHz}$. For each condition we measured the fields for a period of $40 \mathrm{~min}$. The traces shown in Fig. 1-5 are based on 5,000 measurement points between $10 \mathrm{kHz}$ and $5 \mathrm{MHz}$.

For the low frequency range ( $5 \mathrm{~Hz}$ to $32 \mathrm{kHz}$ ), we used the EFA-300 system (Narda Safety Solutions). The magnetic component was measured using the calibrated EFA Magnetic Field Probe $100 \mathrm{~cm}^{2}$ (EFA-300 system, Narda Safety Solutions). The electric component was measured with the calibrated Narda Electric Field Unit (EFA300 system, Narda Safety Solutions). For each measurement, the antennas were connected to the EFA-300 hand held signal analyser, and this signal analyser was also set to 'max hold' and the fields were measured for a period of 40 min (Extended Data Fig. 2).

It must be stressed that anthropogenic electromagnetic noise fields are always present but highly variable in their amplitude, phase and frequency spectrum. Two measurements of their intensity and frequency composition will never be identical. Consequently, the measurements shown in Figs 1c, 1d, 2e, 2f, 5c and 5d are representative examples of the noise measured at the approximate time of day when the experiments were performed. 
The maximal total magnetic field intensity (more precisely the magnetic flux density, $B$ ) in the frequency range between $10 \mathrm{kHz}$ and $5 \mathrm{MHz}$ was calculated using the following equation:

$$
B(\Delta f)=\frac{1}{N} \frac{\Delta f}{\Delta f_{0}} \sum_{i} B_{i}\left(f_{i}, \Delta f_{0}\right)
$$

$B(\Delta f)$ denotes the total magnetic flux density in the bandwidth of interest, $\Delta f=$ $5 \mathrm{MHz}-10 \mathrm{kHz}=4990 \mathrm{kHz}$, and $B_{i}\left(f_{i}, \Delta f_{0}\right)$ are the magnetic flux densities at the $N$ different frequency values $f_{i}$ (every $1 \mathrm{kHz}$ between $10 \mathrm{kHz}$ and $5 \mathrm{MHz}$, i.e. $N=$ 4990) for a resolution bandwidth $\Delta f_{0}$ which equals $10 \mathrm{kHz}$ here. Expressed in words, $B(\Delta f)=($ the sum of the magnetic field intensity values $/ \#$ of values $) \times($ frequency range size / resolution bandwidth), in our case: (the sum of the magnetic field intensity values / 4990 $) \times(4990 \mathrm{kHz} / 10 \mathrm{kHz})$ for the total frequency range from 10 $\mathrm{kHz}$ to $5000 \mathrm{kHz}$. Extended Data Table 1 lists these values for the different conditions tested.

\section{Behavioural experiments.}

All birds were tested in so-called Emlen funnels ${ }^{32}$ lined with scratch-sensitive paper $^{33}$, inside wooden huts ( $4 \mathrm{~m} \times 4 \mathrm{~m} \times$ ca. $3 \mathrm{~m}$, Extended Data Fig. 1a), where no directional cue other than the geomagnetic field was available. In 2005, the experiments took place in these simple wooden huts. After 2007, the walls and ceilings of the huts were lined with aluminium shields as described above. All electronic equipment was placed outside the hut in a separate wooden annex inside an aluminium box and grounded to minimize the generation of electromagnetic noise by the equipment itself.

One hour ( $\pm 10 \mathrm{~min}$ ) before the experiments started (half an hour before until half an hour after sunset), the birds were placed outdoors in wooden transport cages that allowed them to see parts of the evening sky. This gave the birds the possibility to calibrate their magnetic compass from twilight cues ${ }^{17,34}$. Immediately thereafter, the birds were placed in modified aluminium Emlen funnels $(35 \mathrm{~cm}$ diameter, $15 \mathrm{~cm}$ 
high, walls $45^{\circ}$ inclined $^{32}$ ), which were coated with thermal paper ${ }^{33}$ on which the birds left scratches as they moved. The top of each funnel was covered with a translucent Plexiglas lid that prevented the birds from seeing any landmarks in the hut. The overlap point of the paper was adjusted to one of the cardinal directions $(\mathrm{N}$, $\mathrm{S}, \mathrm{E}$ or W). This overlap point was changed randomly between huts and nights. This is important because the papers are always evaluated relative to the overlap point by researchers who do not know in which direction it was positioned. Even if someone would intentionally try to ignore the condition-blinding protocols (this is highly unlikely), this procedure adds a second level of blinding, and it becomes impossible for "wishful thinking" to influence the results in any way, since the persons evaluating the papers cannot know which geographical direction is equivalent to a given direction on the paper. The location of the overlap point is only revealed and taken into consideration, after the primary evaluation of the papers has taken place (for procedures see below).

The birds were tested for one hour under dim white light conditions $\left(2.1 \mathrm{~mW} \mathrm{~m}^{-2}\right)$ produced by incandescent bulbs (spectrum given in reference 12$)$. In each hut, nine birds were tested simultaneously. The birds were placed in a randomized funnel position each night and were put into the funnels from different directions, and we observed no systematic differences between the nine funnel positions or between the four huts. A second, and in a few instances a third, round of tests on a given night started $1.5 \mathrm{~h}( \pm 10 \mathrm{~min})$ after the first or second round. In most cases, each bird was tested in a different hut in each round but under the same magnetic field condition (NMF or CMF) and if applicable under the same timedependent electromagnetic noise condition. The results of the different tests can therefore be treated as independent. The mean direction of each bird in each condition was calculated by unit-vector addition of the individual mean directions from the typically 3-15 tests per bird per condition in which the bird was judged to be oriented.

If more than one condition was tested in a given season, the same experimental birds were tested in all conditions. The experimental condition experienced by a given bird was mostly interchanged every second day, and whenever possible, different conditions were run simultaneously in different huts, so that any putative daily 
variation, for instance induced by the weather ${ }^{35}$, would be averaged out amongst the experimental groups.

In spring 2008, we decided to test the effect of the grounding of the shielding and performed experiments in two different huts. One of them was grounded $(\mathrm{g})$ and the other was left ungrounded $(\mathrm{u})$ without the experimenters knowing which one was which. The experimental condition for each bird alternated every other day; half the birds were tested in g-u-g-u conditions while the other half were u-g-u-g as follows:

\begin{tabular}{|c|c|c|c|c|}
\hline & Days $1 \& 2$ & Days $3 \& 4$ & Days $5 \& 6$ & Days $7 \& 8$ \\
\hline Group 1 & $\mathrm{~g}$ & $\mathrm{u}$ & $\mathrm{g}$ & $\mathrm{u}$ \\
\hline Group 2 & $\mathrm{u}$ & $\mathrm{g}$ & $\mathrm{u}$ & $\mathrm{g}$ \\
\hline
\end{tabular}

The data from these measurements are presented in Fig. 2 as follows:

Fig. 2a

Fig. $2 b$

Fig. 2c

Fig. 2d

$\begin{array}{cccc}\text { Group 1, Days 1 \& } 2 & \text { Group 2, Days 1 \& 2 } & \text { Group 1, Days 5 \& } 6 & \text { Group 2, Days } 5 \& 6 \\ \text { and } & \text { and } & \text { and } & \text { and }\end{array}$

Group 2, Days $3 \& 4 \quad$ Group 1, Days $3 \& 4 \quad$ Group 2, Days $7 \& 8 \quad$ Group 1, Days $7 \& 8$

In 2010 and 2011, we performed experiments in which we added broadband electromagnetic noise (for details see above). The direction of the static magnetic field and electromagnetic noise conditions in a given hut were changed regularly; usually different conditions were tested concurrently in different huts on any given night.

At the rural location, twelve European robins were tested simultaneously in a wooden shelter located in a field (Extended Data Fig. 1c). Here, the birds were tested 
under natural magnetic conditions without a magnetic coil system. Other testing procedures were the same as in the huts on the university campus.

Before we started the experiments in any migratory season, we tested the birds in NMF and CMF conditions with no experimental manipulation for several nights to ensure that they were in migratory mood and to get a control direction.

\section{Orientation data analysis.}

Independently, two researchers visually determined each bird's mean direction to the nearest $10^{\circ}$ from the distribution of the scratches without knowing the direction of the overlap-point of the paper or the magnetic field conditions experienced by the bird. If one of the two researchers considered the scratches to be randomly distributed and the other did not or if the two independently determined mean directions deviated by more than $30^{\circ}$, a third independent researcher was asked to determine the mean direction. If this third individual determined a mean direction similar to one of the first two, and if the individual with initially differing opinion also agreed with this direction, the mean of the two similar directions was recorded as the orientation result. If the three independent researchers could not agree on a mean direction, the bird's heading was defined as random and excluded from the analyses $(7 \%$ of all tests). Birds with fewer than the pre-established lower limit of 100 scratches on the paper were considered inactive ${ }^{15}$ and were also excluded from the analysis (40\% of all tests). The observers performed this screening before they knew the direction of the overlap-point (see above). In this way we can be certain that the person making the decision on whether the bird left more or less than 100 scratches was not influenced by the bird's directional preferences. The average mean heading for each bird was calculated from all its oriented tests recorded under a given experimental condition. Based on these individual mean directions, group mean vectors were calculated by summing unit vectors in the mean directions of each individual bird and dividing by the total number of birds tested. The significance of the group mean vector was tested using the Rayleigh-test ${ }^{36}$. 


\section{Extended Data Figure 1. Wooden huts and experimental locations. a:}

Photograph of one of the four identical wooden huts used for our experiments.

b: Photograph from the inside of an experimental hut showing the aluminium screening, parts of the Merritt coil system, and the table on which the funnels were placed. The insert shows the self-cutting screws used to connect the aluminum plates. c: Simple map of the city of Oldenburg. Built up areas are shown in grey and nature-protected areas in green. Black lines: highways. Blue: water. Red stars: "1" indicates the location of the university campus and "2" the rural location used for some of the tests. d: Map of the University of Oldenburg Wechloy Campus. "1": main university building housing the biology, chemistry, physics and mathematics institutes. "2": botanical greenhouse. "3": iron-free wooden building. "4" the locations of the four wooden huts used for our experiments. "5": "Next Energy" building.

Extended Data Figure 2. Electromagnetic noise measurements in the range from $40 \mathrm{~Hz}$ to $32 \mathrm{kHz}$. a: magnetic field intensity. b: electric field intensity. The colour coding of the traces corresponds to Fig. 4. Notice that the frequency-axis is logarithmic.

Extended Data Table 1. The accumulated time-dependent magnetic field intensity summed over all the frequencies in the spectra recorded for each behavioural test condition.

Additional references for Methods section:

31. Kirschvink, J.L. Uniform magnetic fields and double-wrapped coil systems: improved techniques for the design of bioelectromagnetic experiments. Bioelectromagnetics 13, 401-411 (1991). 
32. Emlen, S.T. \& Emlen, J.T. A technique for recording migratory orientation of captive birds. Auk 83, 361-367 (1966).

33. Mouritsen, H., Feenders, G., Hegemann, A. \& Liedvogel, M. Thermal paper can replace typewriter correction paper in Emlen funnels. J Ornithol 150, 713-715 (2009).

34. Muheim, R., Phillips, J.B. \& Åkesson, S. Polarized light cues underlie compass calibration in migratory songbirds. Science 313, 837-839 (2006).

35: Hein, C. M., Zapka, M. \& Mouritsen, H. (2011) Weather significantly influences the migratory behaviour of night-migratory songbirds tested indoors in orientation cages. J Ornithol 152, 27-35.

36: Batschelet, E. Circular statistics in biology. Academic Press, London (1981). 


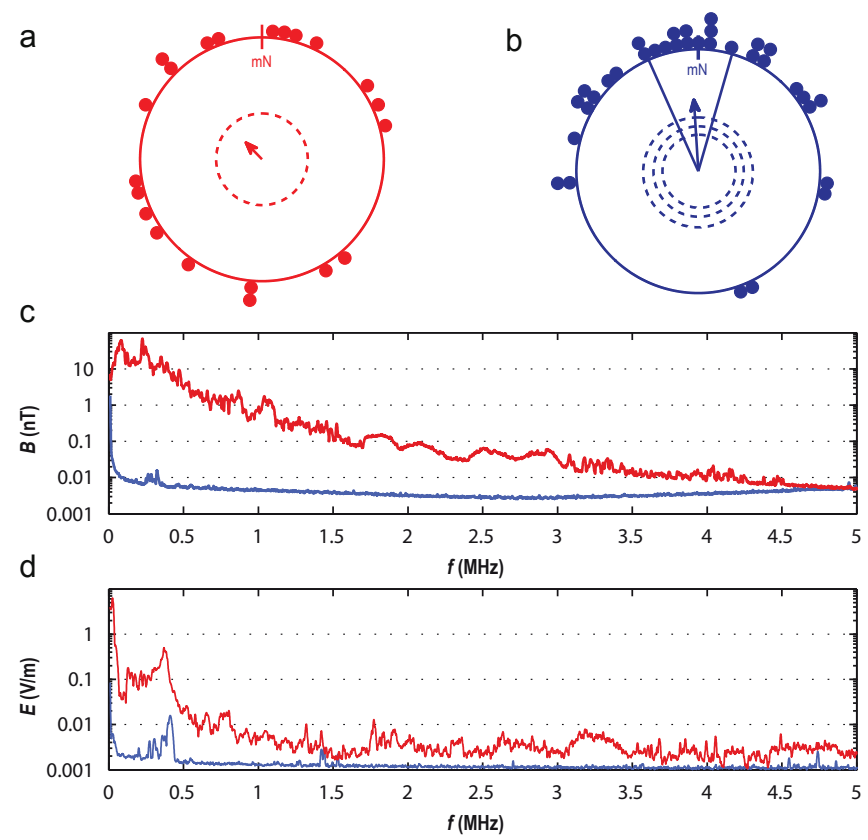



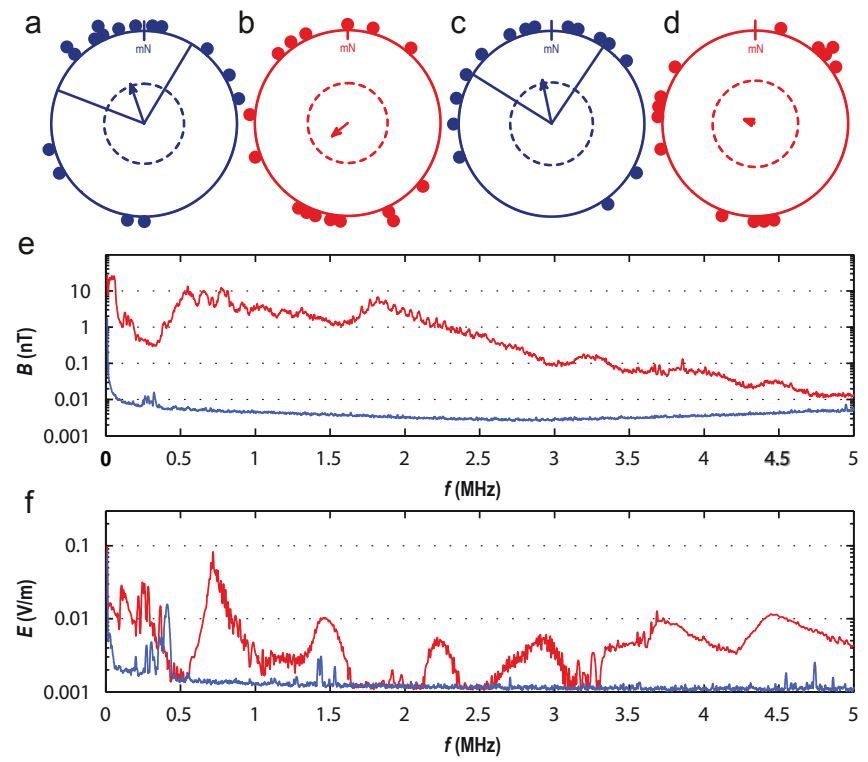

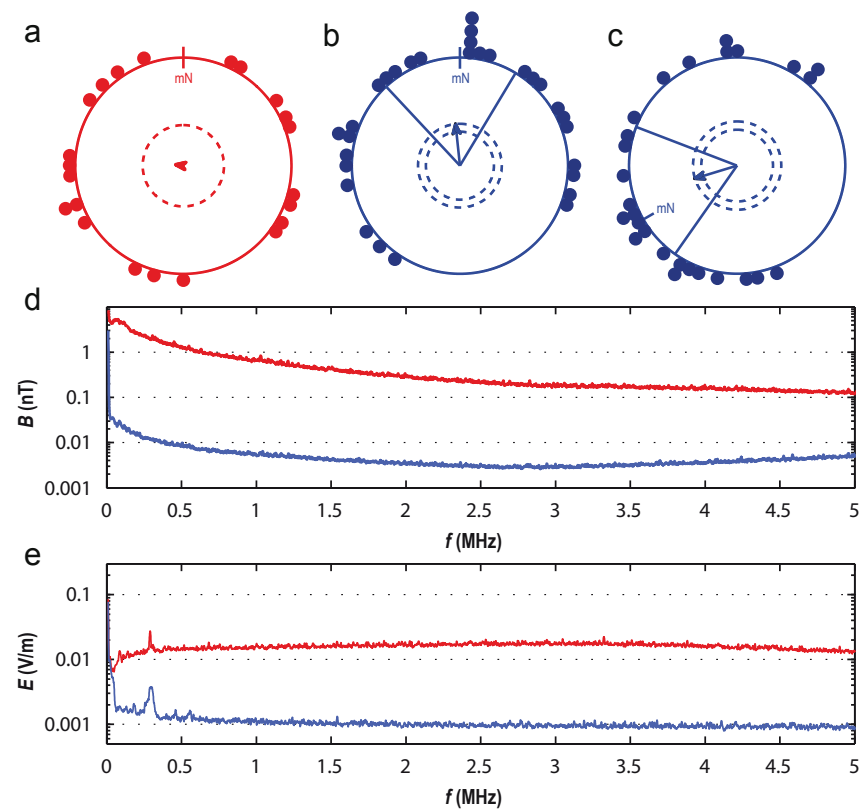

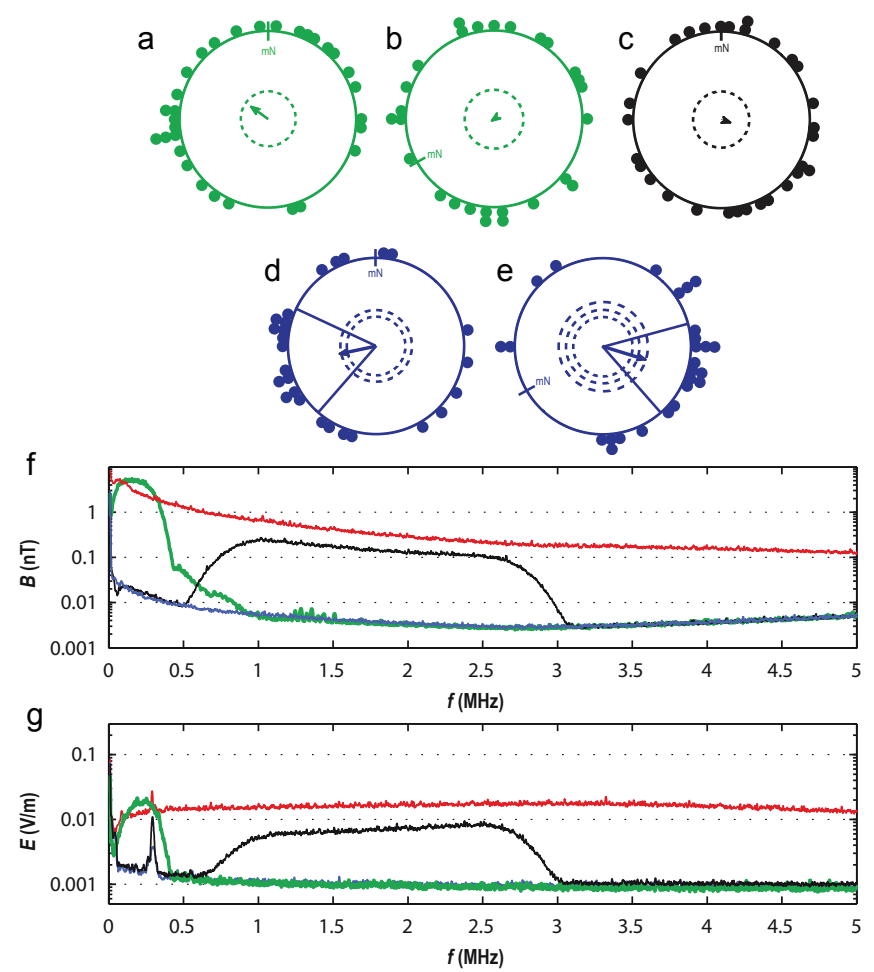
a

a
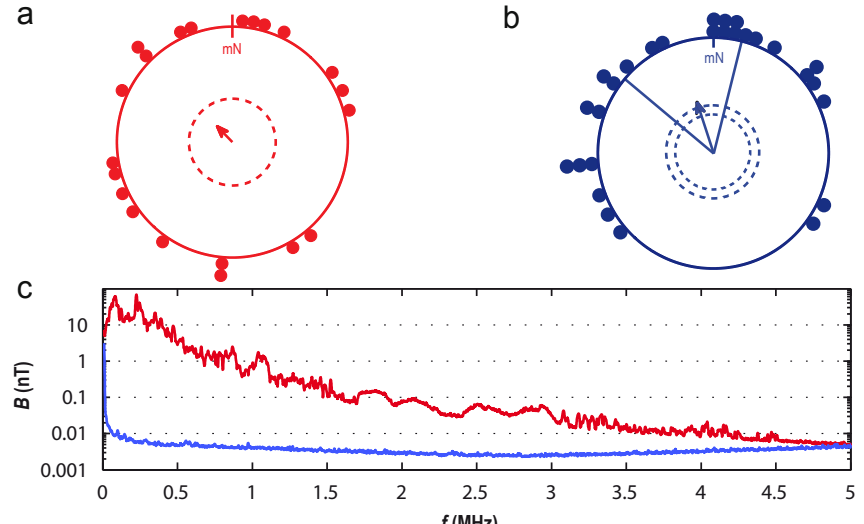

d

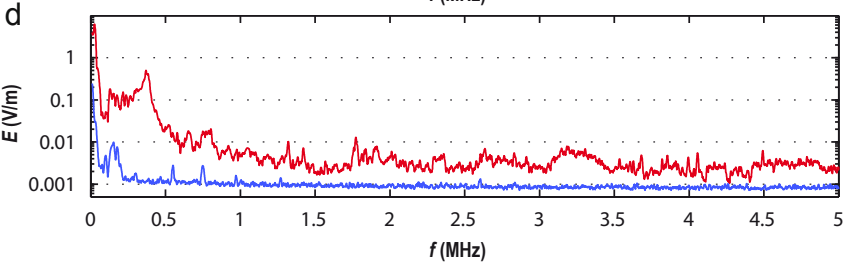

b

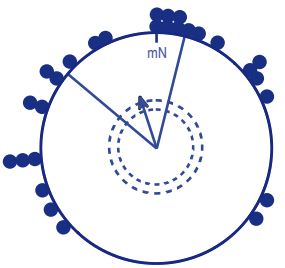

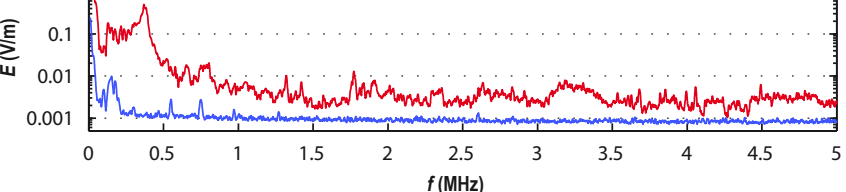




\begin{tabular}{|c|c|c|c|c|c|c|c|c|}
\hline & $\begin{array}{l}\begin{array}{c}\text { Oldenburg } \\
\text { unshielded }\end{array} \\
\text { Fig. } 1 \text { and } 5 \\
\text { red trace }\end{array}$ & $\begin{array}{c}\text { Oldenburg } \\
\text { grounded } \\
\text { shielding } \\
\text { Fig. } 1 \text { and } 2 \\
\text { blue trace }\end{array}$ & $\begin{array}{l}\text { Oldenburg } \\
\text { ungrounded } \\
\text { shielding } \\
\text { Fig. } 2 \text { red } \\
\text { trace }\end{array}$ & $\begin{array}{c}\begin{array}{c}\text { Rural } \\
\text { location }\end{array} \\
\text { Fig. } 5 \\
\text { blue trace }\end{array}$ & $\begin{array}{l}10 \mathrm{kHz}-\mathbf{5} \\
\mathbf{M H z} \\
\text { strong } \\
\text { Fig. } 3 \\
\text { and } 4 \text { red } \\
\text { trace }\end{array}$ & $\begin{array}{c}10 \mathrm{kHz}-5 \\
\text { MHz weak } \\
\begin{array}{c}\text { Fig. } 3 \text { and } 4 \\
\text { blue trace }\end{array}\end{array}$ & $\begin{array}{c}20-450 \\
\text { kHz } \\
\text { bandpass } \\
\text { Fig. } 4 \\
\text { green } \\
\text { trace }\end{array}$ & $\begin{array}{c}600 \mathrm{kHz}-3 \\
\mathrm{MHz} \\
\text { bandpass } \\
\text { Fig. } 4 \text { black } \\
\text { trace }\end{array}$ \\
\hline$f(\mathbf{k H z})$ & \multicolumn{8}{|c|}{ Accumulated field intensity (nT) } \\
\hline $\begin{array}{c}10- \\
5000\end{array}$ & 1008.10 & 2.56 & 827.86 & 2.60 & 278.88 & 3.31 & 133.09 & 34.83 \\
\hline $\begin{array}{l}100- \\
5000\end{array}$ & 714.17 & 1.98 & 705.88 & 1.74 & 229.56 & 2.35 & 101.11 & 33.98 \\
\hline $\begin{array}{c}20- \\
450\end{array}$ & 855.63 & 0.38 & 125.59 & 0.31 & 119.30 & 0.69 & 128.27 & 0.71 \\
\hline $\begin{array}{l}600- \\
3000\end{array}$ & 81.89 & 0.87 & 561.05 & 0.79 & 98.81 & 1.00 & 1.16 & 32.48 \\
\hline
\end{tabular}




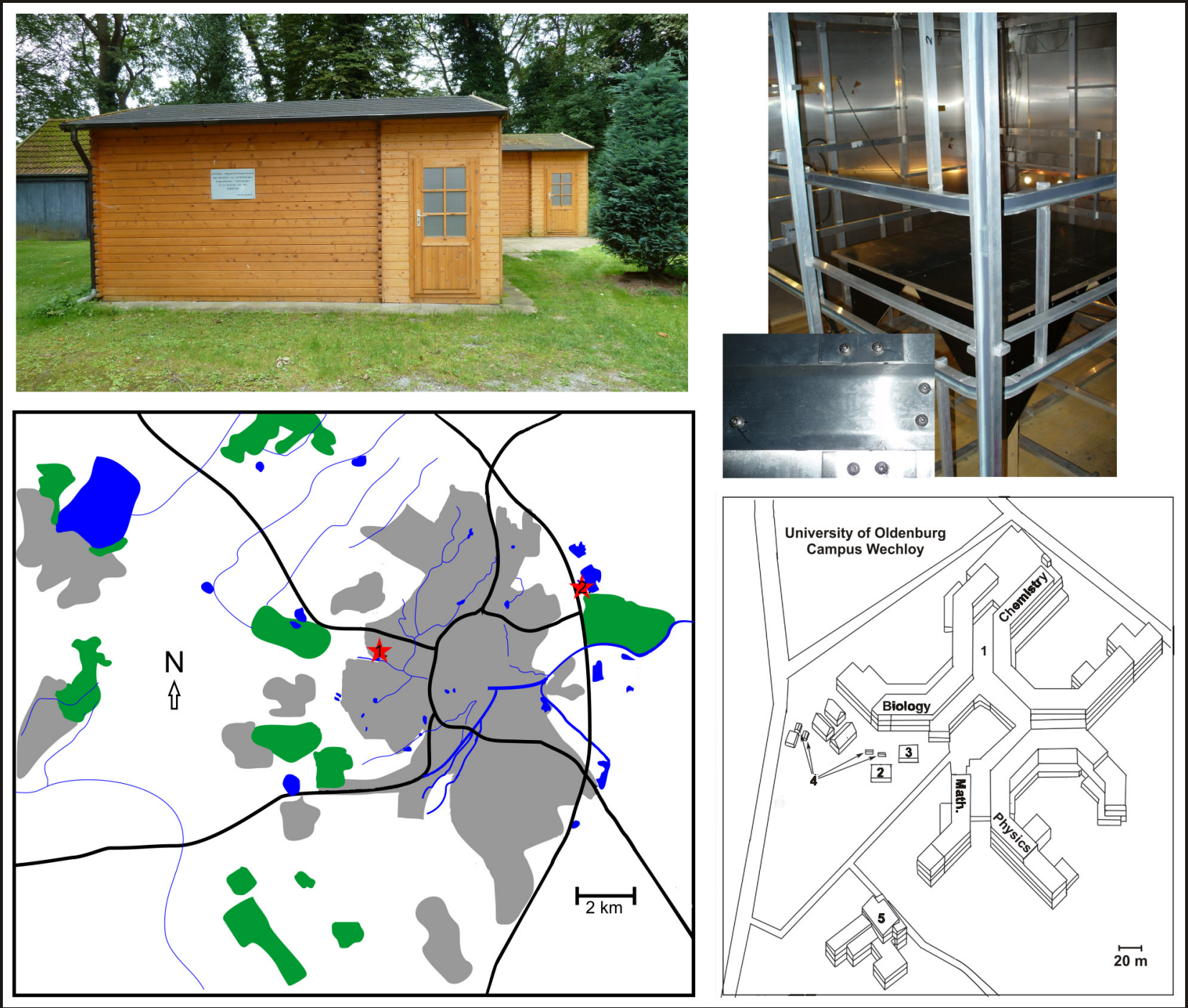



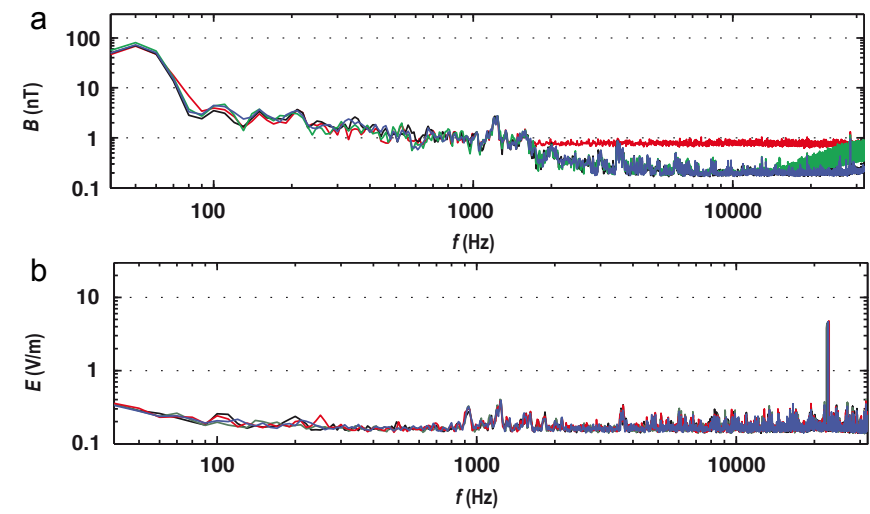

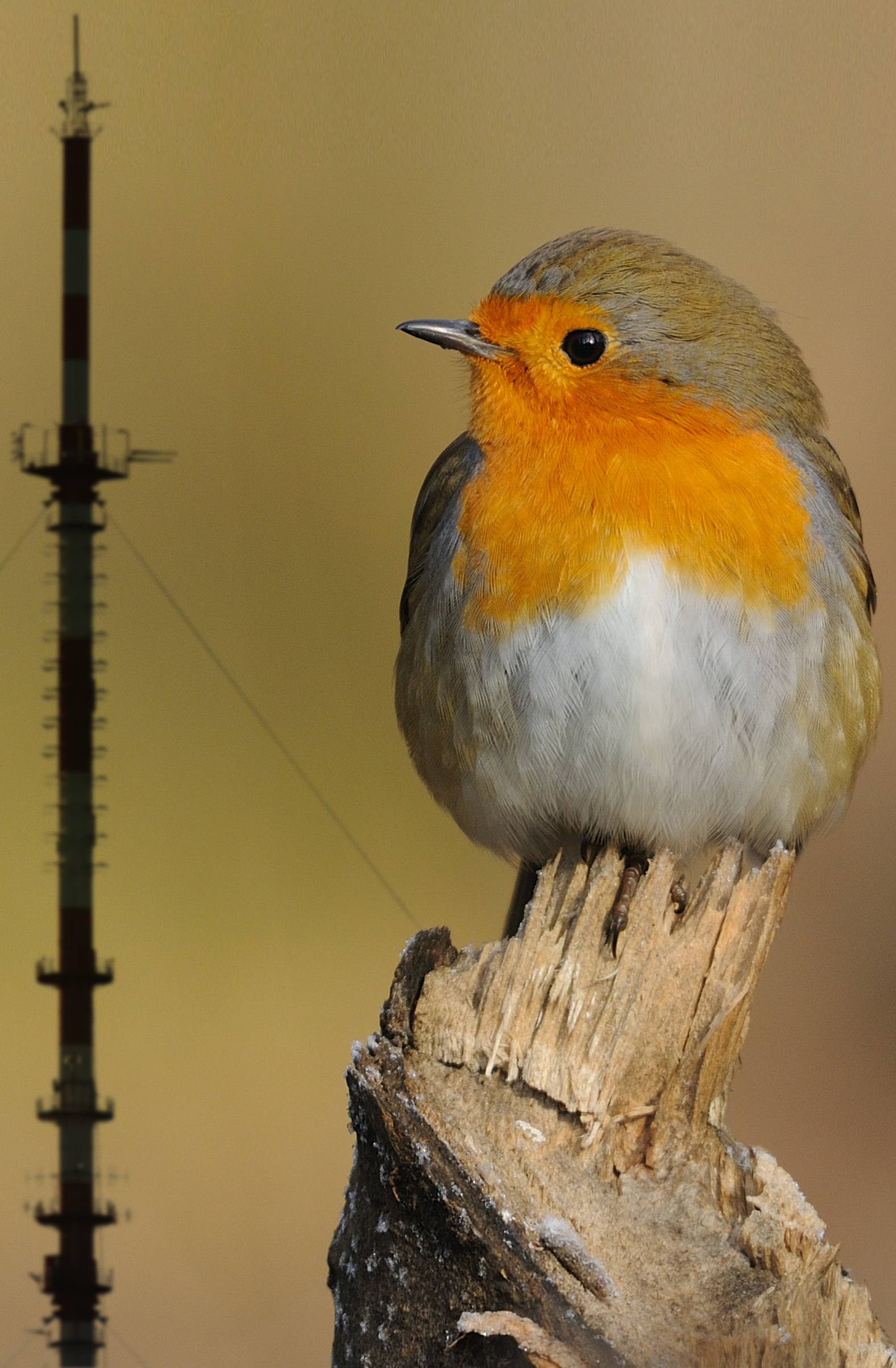